Revue d'histoire de l'Amérique française

DEV REVE D.HISTOIRE DE L'AMÉRIQUE FRANÇAISE

\title{
Les alliances matrimoniales exogames dans le gouvernement de Québec - 1700-1760
}

\author{
Jacques Mathieu, Céline Cyr, Guy Dinel, Jeannine Pozzo et Jacques St-Pierre
}

Volume 35, numéro 1, juin 1981

URI : https://id.erudit.org/iderudit/303925ar

DOI : https://doi.org/10.7202/303925ar

Aller au sommaire du numéro

Éditeur(s)

Institut d'histoire de l'Amérique française

ISSN

0035-2357 (imprimé)

1492-1383 (numérique)

Découvrir la revue

Citer cet article

Mathieu, J., Cyr, C., Dinel, G., Pozzo, J. \& St-Pierre, J. (1981). Les alliances

matrimoniales exogames dans le gouvernement de Québec - 1700-1760. Revue

d'histoire de l'Amérique française, 35(1), 3-32. https://doi.org/10.7202/303925ar d'utilisation que vous pouvez consulter en ligne. 


\title{
LES ALLIANCES MATRIMONIALES EXOGAMES DANS LE GOUVERNEMENT DE QUÉBEC 1700-1760*
}

Département d'histoire

Université Laval

\author{
JACQUES MATHIEU \\ CÉLINE CYR \\ GUY DINEL \\ JEANNINE POZZO \\ JACQUES ST-PIERRE
}

Depuis quelques années, un nombre croissant d'enquêtes scientifiques menées par des anthropologues, démographes, ethnologues, géographes, sociologues et historiens convergent vers la famille et cela en vue d'une meilleure connaissance des règles et des pratiques sociales. Les choix d'un conjoint, d'une profession, d'un lieu d'établissement, d'un héritier sont vus comme autant de stratégies familiales pour transmettre un patrimoine, une culture, un savoir, un statut social.

Notre recherche s'inscrit dans cette perspective large visant d'abord à identifier puis à expliquer les pratiques sociales en milieu rural. La première phase consiste à préciser l'importance du phénomène des alliances matrimoniales exogames, soit celles comportant un étranger à la paroisse - et parfois deux - lors de la célébration du mariage. Il s'agit là d'un premier niveau d'étude, où les dimensions géographiques sont privilégiées par rapport aux aspects sociaux, professionnels ou culturels de l'endogamie. Nous avons ensuite étudié les mariages pour repérer l'existence d'attitudes et de comportements révélateurs de stratégies. L'évolution de l'importance numérique des étrangers, la précision de leur provenance et l'identification des facteurs favorables à leur présence permettent, dans un premier temps, d'évaluer jusqu'à quel point la paroisse rurale constitue, au XVIIIe siècle, un monde ouvert ou fermé.

* Cette étude s'inscrit dans le cadre des préoccupations du groupe de recherche sur les sociétés rurales comparées de la France de l'Ouest et du Québec. Cependant notre comparaison comporte trois lacunes: nous n'avons conduit aucune recherche personnelle en France, nos connaissances se limitent aux études publiées qui ne rejoignent pas nécessairement nos objectifs. 
Pour étudier ce phénomène, nous avons choisi cinq paroisses du gouvernement de Québec: Charlesbourg et Neuville sur la rive nord, Beaumont, Saint-Nicolas et Rivière-Ouelle sur la rive sud. Ce choix, fondé sur les intérêts de chacun, satisfaisait également les exigences de la recherche. Les dates de concession, les bassins de population, les étapes du développement, leur localisation dans des régions différentes et la proximité ou l'éloignement de la ville étaient assez variés pour donner des résultats représentatifs qu'une comparaison rendait significatifs. L'enquête porte sur la période 1700-1760, une époque où la population a atteint une certaine stabilité et se renouvelle essentiellement grâce aux gens nés sur place.

Une fois le choix des paroisses arrêté, nous avons dépouillé systématiquement les registres d'état civil conservés aux Archives nationales du Québec. La compilation des baptêmes, mariages et sépultures a fourni un corpus d'environ 15000 actes dont plus de 1500 mariages. Le relevé de ces derniers a été réalisé suivant le modèle des fiches de compilation élaboré par Henry et Fleury, où sont notés la date et le lieu de célébration du mariage, les noms des conjoints et des parents, le lieu de résidence, le statut civil des conjoints et des parents, l'âge et la profession des conjoints, les dispenses de bans et l'aptitude à signer des conjoints ${ }^{1}$.

Les lacunes dans l'enregistrement sont parfois totales. Les registres ne comportent aucune inscription pendant un ou plusieurs mois consécutifs comme c'est le cas à Beaumont du mois d'août à la fin de l'année de 1727 et en 1757, à Charlesbourg, en février et mars 1738 et en décembre 1749, à Rivière-Ouelle, en 1705 et 1715 . Des registres mal tenus, une succession rapide de curés, un prêtre malade, remplacé à l'occasion par un missionnaire, ou occupé à desservir une paroisse voisine, entraînant un sous-enregistrement constant, mais dilué dans le temps, donc plus difficile à saisir $^{2}$. La guerre de la Conquête laisse des traces partout: à Neuville, le curé inscrit deux ou trois décès dans le même acte; les Britanniques occupent Beaumont à compter de juin 1759. Par ailleurs, les curés attachent manifestement plus d'attention à inscrire les mariages que les baptêmes ou les sépultures. Les cas de nouveau-nés ondoyés à leur naissance et conduits à l'église parfois bien plus tard influencent aussi notablement l'inscription des

1 Michel Fleury et Louis Henry, Nouveau manuel de dépouillement et d'exploitation de l'état civil ancien (Institut national d'études démographiques, Paris, 1965).

2 L'on trouvera une analyse critique détaillée de ces sources dans Hubert Charbonneau, Vie et mort de nos ancêtres. Étude démographique (Montréal, P.U.M. 1975), 69-114. 
naissances ${ }^{3}$. L'enregistrement de seulement trois mortalités infantiles durant la décennie 1700-1709 en regard des 57 baptêmes donne une moyenne inacceptable. L'immensité du territoire et l'existence de dessertes affectent également la qualité de l'enregistrement. Jusqu'en 1710, le curé de Rivière-Ouelle doit parcourir 100 kilomètres pour aller d'une extrémité à l'autre de sa paroisse. Quand un prêtre dessert une autre paroisse, les actes sont parfois mêlés; ceux de Saint-Michel de La Durantaye se retrouvent dans les registres de Beaumont entre 1705 et 1726 . Le curé qui desservait Saint-Antoine-de-Tilly et Saint-Nicolas a parfois inscrit au registre de l'une des actes relatifs à l'autre. La subdivision de paroisses a aussi eu pour effet de réduire considérablement le nombre d'actes consignés. Â l'origine, Rivière-Ouelle couvrait le territoire compris entre Saint-Roch-des-Aulnaies et Rivière-du-Loup. Elle est amputée des secteurs Kamouraska - Rivière-du-Loup en 1709 et Saint-Roch - Sainte-Anne en 1715. La paroisse de Beaumont est divisée en deux en 1748: Saint-Étienne revenant aux limites originales de la seigneurie, tandis que l'augmentation concédée en 1713 forme la nouvelle paroisse de Saint-Charles.

D'un autre côté, la lecture du document, le relevé des informations et le traitement des données influencent également la nature des résultats. Nous avons relevé l'âge réel au décès en sachant fort bien que certains faisaient des approximations et se permettaient parfois quelques fantaisies. Il nous semblait cependant que la traditionnelle répartition en deux grands groupes, mortalité infantile et autres, restait insuffisante pour évaluer le phénomène des remariages. De fait, il est apparu manifeste que les épidémies touchaient des groupes d'âge particulier. Rarement a-t-on vu la mort faucher indistinctement des gens de tous âges. Bien au contraire. Les épidémies frappaient pendant quelques mois, à un endroit, soit des bébés, soit des enfants entre 1 et 15 ans, soit des adultes ou encore des gens de 50 ans et plus, affectant différemment la courbe des naissances et des mariages.

Le relevé des mariages posait plus de problèmes. Nous avons écarté le traitement systématique des informations relatives aux parents défunts parce que l'enregistrement était souvent incomplet. La mention du statut de veuf ou de veuve paraît valable; rarement une personne va-t-elle taire cette situation. Il est aussi possi-

3 Il y a plusieurs cas semblables, en particulier dans la paroisse de Rivière-Ouelle, vraisemblablement à cause de son étendue. Le cas de Pierre Demers né à Saint-Nicolas en 1733, «légitimement baptisé par Monsieur Roullard pour lors missionnaire» et «n'ayant point été présenté ny apporté à l'église pour recevoir les cérémonies accoutumées dans le baptême," et auxquelles le curé a suppléé le 6 avril 1750 est aussi révélateur. ANQ, Q, Registres d'état civil, Saint-Nicolas, 6 avril 1750. 
ble que les dispenses, surtout dans les cas de consanguinité pour une parenté éloignée, n'aient pas été demandées ou consignées au registre, mais plusieurs indices portent à croire que de telles omissions aient été assez rares.

Nous avons considéré comme "étranger» les personnes identifiées par un lieu de résidence extérieur à la paroisse, par une mention comme «auparavant domicilié à» ou «depuis 6 mois dans la paroisse» ou encore dont les bans étaient publiés ailleurs. De telles mentions indiquent clairement l'état et la perception d'étranger. De plus, pour éviter de gonfler artificiellement le nombre d'étrangers, nous avons écarté les couples venus d'une paroisse voisine à cause de l'absence ou de la maladie de leur curé ${ }^{\text {. }}$.

L'importance numérique des conjoints étrangers et leur répartition dans l'espace révèlent d'importantes différences de comportement entre la situation en France et en Nouvelle-France. Diverses coupes dans le temps permettent d'évaluer le poids de conditions locales de développement. Aussi cette analyse débouche-t-elle sur l'influence respective des circonstances immédiates comme les épidémies ou la guerre ou de situations telles le veuvage, la colonisation et les liens familiaux.

\section{Importance numérique des conjoints étrangers au mariage}

Il existe plusieurs façons de prendre la mesure des phénomènes d'exogamie et d'endogamie dans la société rurale d'Ancien Régime. Le pourcentage d'endogamie est basé le plus souvent sur le rapport entre le nombre d'étrangers et celui des résidents ${ }^{5}$. Cette mesure, pour satisfaisante qu'elle soit sur certains plans, reflète assez mal la réalité sociale que l'on veut saisir. Il est tout à fait exceptionnel que le mariage soit célébré à l'extérieur du lieu de résidence d'au moins un des époux. Le calcul de l'endogamie commence alors avec un avantage de l'ordre de $50 \%{ }^{6}$. En plus, selon

4 Ces cas sont exceptionnels et assez rares. Il s'agit néanmoins d'alliances endogames célébrées hors de la paroisse de résidence des deux conjoints.

5 Procèdent de cette façon entre autres: Martine Ségalen, Nuptialité et alliance dans une commune de l'Eure (Paris, Maisonneuve et Larose, 1972), 89-93; P.J. Perry, «Mariage et distance dans le canton du Bleymard (Lozère) 1811-1820 et 1891-1900», Études rurales (juil.-sept. 1977): 63; Jacqueline Vu Tien Khang et André Sévin, Choix du conjoint et patrimoine génétique. Étude de quatre villages du Pays de Sault de 1740 à nos jours (Paris, CNRS, 1977), 131; Louise Dechêne, Habitants et marchands de Montréal au XVIIe siècle (Paris, Plon, 1974), 104.

Par contre, André Burgières, Bretons de Plozénet (Paris, Flammarion, 1975), 43-44, considère comme «union exogame tout mariage dont l'un des conjoints n'est pas originaire de Plozénet»; René Jetté, "La stratification sociale: une direction de recherche», RHAF, 26, 1 (juin 1972): 40, base aussi le taux d'endogamie sur le «rapport entre le nombre de mariages..."

6 Il ne fait pas de doute que la définition et le calcul de l'endogamie posent un problème méthodologique dans la mesure où les définitions utilisées diffèrent considérablement. 
les prescriptions canoniques, le mariage doit être célébré dans la paroisse de la future mariée. Cette règle semble avoir été respectée de façon très stricte en Nouvelle-France. De fait, dans les cinq paroisses étudiées, les femmes ne représentent que $12,3 \%$ des conjoints étrangers. Les déboires d'une jeune veuve de l'AncienneLorette - même s'ils datent de 1768 - sont révélateurs de cette intransigeance religieuse ${ }^{7}$. En raison de son entêtement à vouloir se remarier dans la paroisse de son futur époux, elle fut excommuniée avec tous ceux qui l'avaient soutenue. En France, l'application de cette règle semble avoir été plus souple comme l'indique la proportion d'étrangers féminins: à Meulan entre 1690 et $1789,40 \%$ des épouses viennent de l'extérieur; dans le Pays de Sault, seulement trois jeunes filles sur quatre se marient dans leur paroisse de résidence ${ }^{8}$. Ainsi les pourcentages d'exogamie calculés en France comprennent une proportion élevée de femmes et rendent les comparaisons difficiles, sinon impossibles.

Ces différentes raisons nous ont fait opter pour une évaluation des alliances exogames plutôt que de considérer les individus. L'objectif est d'établir quelle proportion de mariages comporte une alliance avec un étranger à la paroisse. Entre 1700 et 1760 , 1531 mariages ont été célébrés dans les cinq paroisses étudiées. De ce nombre, 642 soit $41,9 \%$ des mariages comprenaient au moins un conjoint étranger à la paroisse. C'est une proportion qui nous paraît très élevée.

\section{Découpage territorial et provenance des conjoints étrangers}

En France, des études extrêmement poussées, reposant sur une mesure au kilomètre près, ont abouti à des conclusions solidement argumentées mettant en évidence l'importance de l'espace géographique dans le choix du conjoint. L'immensité du territoire de la Nouvelle-France et l'état de son développement créent cependant un contexte très différent; d'où l'intérêt d'évaluer la pertinence de ce facteur sur le choix d'un conjoint et de préciser les règles de pratiques exogames.

De part et d'autre, la préoccupation première est la même. Il s'agit de déterminer, à partir des registres d'état civil, dans quelle mesure une communauté est repliée sur elle-même ou ouverte à l'extérieur. Les enquêtes menées en divers points du territoire français indiquent que l'aire géographique dans laquelle se choisissent les conjoints est extrêmement restreinte. À Ribennes en Gévaudan, par exemple, $87 \%$ des mariages célébrés entre 1650 et 1830 ont été

7 Louis Allard, L'Ancienne-Lorette (Montréal, Leméac, 1979), 143-144.

8 Ségalen, op. cit., 93 et Vu Tien Khang et Sévin, op. cit., 36. 
conclus entre des individus vivant à moins de 15 kilomètres ${ }^{9}$. Dans le canton du Bleymard, au début du XIXe siècle, 97,5\% des alliances unissaient des conjoints résidant dans un rayon de 20 kilomètres ${ }^{10}$. Par corrélation et régression, l'auteur de cette dernière étude montre que $92 \%$ des mariages s'expliquent par le facteur distance. Pour lui, des contacts réguliers et fréquents - indispensables en vue d'une alliance - n'étaient possibles que si la distance à parcourir depuis le domicile pouvait être franchie à pied. Le relief du territoire concerné prend alors une grande importance facilitant ou limitant les déplacements ${ }^{11}$.

Jacqueline Vu Tien Khang, qui a étudié l'évolution du patrimoine génétique de quatre villages des Pyrénées de 1740 à nos jours, retient des critères semblables. Selon elle: «de toutes les contraintes qui pèsent sur le choix du conjoint, la plus évidente est de nature géographique: la possibilité de choix est en effet conditionnée par la possibilité de rencontre». Mais la distance n'est pas seule en cause, «interviennent également de façon déterminante les contraintes imposées par le relief qui influent sur les possibilités de communication ${ }^{12}{ }^{12}$. C'est pourquoi elle convertit la distance en temps, soit une heure de marche, d'où une certaine fréquence des alliances entre résidents de villages voisins. Elle conclut son analyse en ces termes: «En résumé, on peut dire qu'on se marie sur place plutôt que dans le village immédiatement voisin: quand on se marie dans un village voisin, on choisit de préférence le plus proche. $\gg{ }^{13}$ Malgré la cohésion très forte de la communauté, le tissu des relations parentales dépasse les strictes limites du village.

En Nouvelle-France, le facteur distance ne peut jouer de la même façon et la cartographie des alliances matrimoniales diffère passablement de l'exemple français. À l'exception de Charlesbourg, située près de la ville de Québec et où il serait possible de constituer une forme d'aires concentriques ${ }^{14}$, le peuplement s'étire en un long ruban linéaire sur les deux rives du Saint-Laurent. À raison d'un établissement tous les 180 mètres, le choix d'un conjoint se trouve confiné à une trentaine de familles dans la limite des cinq kilomètres, à une soixantaine dans l'entité territoriale de base.

9 Pierre Lamaison, «Les stratégies matrimoniales dans un système complexe de parenté: Ribennes en Gévaudan (1650-1830)», Annales Économies Sociétés Civilisations, 34, 4 (juil.-août 1979): 729.

10 Perry, op cit., 64.

11 Ibid.

$12 \mathrm{Vu}$ Tien Khang et Sévin, op. cit., 55.

13 Ibid., 61.

14 La paroisse de Charlesbourg est constituée d'une dizaine de bourgs ceinturant deux villages où les terres sont divisées en étoile. Il n'a pas été possible d'étudier les échanges entre les différentes parties de la paroisse, les curés n'ayant pas toujours précisé le lieu exact de résidence des conjoints. 
Ainsi un mariage endogame peut représenter un éloignement de dix kilomètres, soit un parcours d'une durée de deux heures, tandis qu'une alliance exogame peut associer des jeunes gens dont les parents sont voisins ou presque. Par ailleurs, les obstacles naturels, qui sont à l'échelle du territoire, ne représentent jamais des barrières infranchissables. Les Canadiens sont habitués à affronter le fleuve, les rigueurs de l'hiver et l'isolement. Règle générale, la topographie locale n'a qu'une influence limitée.

Les fréquentations se déroulent aussi dans un contexte différent. Les circonstances, tels bals ou fêtes, favorisant la rencontre de jeunes gens, dont l'attirance réciproque pourrait aller à l'encontre des règles sociales et des traditions, sont rarissimes ${ }^{15}$. En l'absence de villages - les premiers sont constitués en 1757 - et à défaut d'un marché local, l'assistance aux cérémonies religieuses devient l'occasion privilégiée des rencontres. Ce climat s'avérait fort peu favorable au relâchement de l'emprise familiale dans le choix du conjoint ${ }^{16}$.

L'analyse géographique de l'exogamie prend en NouvelleFrance une orientation bien particulière. Elle est plus significative à grande échelle que dans la mise en relation de points dans l'espace. Aussi avons-nous procédé à un découpage territorial afin de mieux faire ressortir certaines particularités des alliances exogames.

Les institutions coloniales ont fortement contribué à déterminer les entités territoriales. L'instauration du régime royal, en 1663, a entraîné la formation de trois gouvernements. Le régime seigneurial et le cadre paroissial ont, de leur côté, amené la constitution de petites localités bien identifiées, dont les frontières étaient souvent identiques ${ }^{17}$.

Ce découpage ne reflète toutefois qu'une partie de la géographie canadienne et n'éclaire qu'imparfaitement les caractères géographiques de la formation des alliances matrimoniales. Entre le gouvernement de Québec qui couvre les deux rives du fleuve et s'étend sur environ 300 kilomètres et la seigneurie qui dépasse rarement 15 kilomètres de front, une autre réalité se dégage: la région.

15 Selon Robert-Lionel Séguin, Les divertissements en Nouvelle-France (Ottawa, 1968), 21-22, les veillées, toujours organisées par des adultes, sont d'abord des réunions de famille.

i6 Sur l'importance de l'emprise familiale, voir notamment, Ségalen, Mari et femme dans la société paysanne (Paris, Flammarion, 1980), $214 \mathrm{p}$.

17 Hubert Charbonneau et Yolande Lavoie, "Cartographie du premier découpage territorial des paroisses du Québec, 1721-1722», La Revue de géographie de Montréal, XXVII, 1 (1973): 81-87. 
Pour l'ensemble du gouvernement de Québec, nous avons reconstitué dix régions. Il s'agit plus précisément de: Charlevoix, la côte de Beaupré, l'île d'Orléans, les environs de Québec (depuis le saut de Montmorency jusqu'à la rivière du Cap Rouge), Portneuf (depuis la rivière du Cap Rouge jusqu'au gouvernement de Trois-Rivières), Lotbinière (depuis le gouvernement de TroisRivières jusqu'au saut de la Chaudière), Lévis, considérée comme une région distincte à cause surtout de sa situation particulière par rapport à Québec, la côte du Sud (depuis Beaumont jusqu'à St-Jean-Port-Joli inclusivement), Kamouraska (depuis SaintRoch-des-Aulnaies jusqu'à Rivière-du-Loup), enfin la Beauce, qui ne s'ouvre à la colonisation que dans le second quart du XVIIIe siècle.

Chaque région ainsi définie correspond à un espace délimité par des frontières naturelles et à une réalité physique perçue par les autorités même si elles n'ont jamais été juridiquement constituées. D'une part, les rivières ont longtemps représenté les barrières les plus importantes à la circulation des hommes ${ }^{18}$. Les rivières Montmorency, du Cap Rouge et Chaudière, ont donc servi de frontière. Les ressources propres à la région ont aussi contribué à engendrer des activités particulières en plus de l'agriculture ${ }^{19}$. D'autre part, les contraintes géographiques se sont nettement reflétées dans les commissions et les aires de pratique des notaires ${ }^{20}$ et dans le processus d'élaboration des recensements entre 1685 et $1739^{21}$. Les recoupements et les réunions de seigneuries lors des recensements montrent bien la transposition au plan institutionnel des impératifs géographiques. C'est pourquoi aussi le fleuve représente une barrière entre les régions.

Ainsi nous estimons que la région correspond à un espace vécu. De plus, ce type de découpage permettait d'analyser de façon plus significative la provenance de conjoints étrangers. D'une part, il reflétait mieux l'éloignement d'un conjoint venant de l'extérieur. D'autre part, il permettait de comprendre que les habitants

18 Les procès-verbaux du procureur général Collet en vue de l'établissement des limites paroissiales (RAPQ (1921-22): 262-380) contiennent de nombreuses plaintes de la part d'habitants qui devaient traverser une rivière pour se rendre à l'église.

19 Voir, par exemple, le «Mémoire de Gédéon de Catalogne sur les plans des seigneuries et habitations des gouvernements de Québec, les Trois-Rivières et Montréal», Bulletin des recherches historiques, 21, 11 (nov. 1915): 321-335.

20 Ce découpage des aires de pratique est particulièrement manifeste dans les régions rurales colonisées à la fin du XVIIle et au début du XVIIIe siècle. Joseph-Edmond Roy, Histoire du notariat au Canada depuis la fondation de la colonie jusqu'à nos jours, (Lévis 1899) vol. 1: 161-162.

21 Les regroupements successifs et variés des localités rencensées illustrent particulièrement bien ce phénomène. AC, Série G1, vol. 460-461. Sur la confection des recensements, voir aussi, Guy Frégault, «La Nouvelle-France: territoire et population», Le XVIIIe siècle canadien (Montréal, HMH, 1968): 33-34 et Louise Dechêne, La correspondance de Vauban relative au Canada (Québec, Ministère des Affaires culturelles, 1968), 4-5. 
de Saint-Nicolas, située à l'extrémité est de la région de Lotbinière, aient entretenu plus de contacts avec cette région qu'avec les paroisses situées de l'autre côté de la rivière Chaudière.

Les étrangers ont été répartis en cinq catégories, selon qu'ils provenaient des paroisses limitrophes, de la même région, d'autres régions du gouvernement de Québec, des autres gouvernements, ou de l'extérieur de la colonie (voir annexe I). Toutefois, une répartition simplifiée, basée sur l'éloignement ou la proximité des conjoints, s'est avérée plus pertinente.

\section{TABLEAU I}

Répartition en pourcentage de la provenance des conjoints étrangers

Beaumont

Charlesbourg

Neuville

Rivière-Ouelle

Saint-Nicolas

Total
Même région

34,8

41,1

33,6

53,9

47,1

41,8
Localité éloignée*

65,2

58,9

66,4

46,1

49,4

58,2

* - Par localité éloignée, il faut entendre autres régions du gouvernement de Québec, autres gouvernements et extérieur de la colonie.

- On retrouve à Saint-Nicolas trois conjoints étrangers dont l'origine reste inconnue.

Le constat majeur de cette analyse tient au fait que la majorité des conjoints étrangers (389 sur 665 , soit $58,2 \%$ ) viennent d'une localité éloignée. Si l'on reporte ces données sur l'ensemble des mariages, un conjoint sur quatre $(25,3 \%)$ va chercher un partenaire loin de son lieu de résidence. Dans le canton du Bleymard, au début du XIXe siècle, le pourcentage des conjoints résidant à plus de 20 kilomètres est dix fois moindre, soit $2,5 \%$.

Pour rendre plus adéquate la comparaison avec la situation en France, il peut être utile de dégager la part du fait colonial. Ainsi $19,1 \%$ de tous les conjoints étrangers (127 sur 665) viennent de la mère patrie. C'est trop peu pour modifier fondamentalement le rapport entre l'éloignement et la proximité. Par contre, l'établissement d'immigrants dans le monde rural paraît d'une importance numérique non négligeable d'autant plus que ces étrangers se dis- 
tribuent très inégalement ${ }^{22}$. Ils s'établissent plus facilement à proximité de la ville ou aux frontières de la colonie, Saint-Nicolas faisant exception. La politique de peuplement des autorités, dont l'hébergement des soldats, peut contribuer à expliquer cette répartition.

À un degré moindre, la faible mobilité de la ville vers la campagne et d'un gouvernement à un autre est à souligner. Seulement $14,4 \%$ de tous les conjoints étrangers (96 sur 665) viennent de la ville, soit passablement moins que le nombre d'immigrants. Et, si l'on exclut la paroisse de Charlesbourg située près de Québec, moins de $3 \%$ de tous les mariages en milieu rural concernent des conjoints venant de la ville ${ }^{23}$. Les déplacements d'un gouvernement à un autre sont encore moins nombreux (22 sur 665) dont dix pour la seule paroisse de Neuville, située plus au coeur de la colonie.

Dans les paroisses étudiées, le conjoint étranger est généralement un homme, un rural, résident du gouvernement de Québec et qui peut venir de n'importe quelle région.

La plus importante région pourvoyeuse de conjoints étrangers est toujours celle dont fait partie la paroisse, mais il en vient de partout ${ }^{24}$. Les chiffres montrent aussi un mouvement de population allant des régions les plus anciennement peuplées vers celles plus récemment ouvertes à la colonisation, mais l'inverse se produit également. Enfin, certaines localités éloignées fournissent de forts contingents de conjoints, ce qui laisse présumer de l'existence d'autres liens, notamment familiaux.

22 La répartition de ces conjoints se conçoit sur deux bases différentes: par rapport à l'ensemble des immigrants repérés et quant à la proportion qu'ils constituent dans une paroisse.

$\begin{array}{lccc}\text { Beaumont } & \begin{array}{c}\text { Nombre } \\ \text { d'immigrants } \\ \text { français }\end{array} & \begin{array}{c}\text { \% du nombre } \\ \text { d'immigrants }\end{array} & \begin{array}{c}\text { \% de conjoints } \\ \text { étrangers }\end{array} \\ \text { Charlesbourg } & 10 & 7,9 & 8,9 \\ \text { Neuville } & 73 & 57,4 & 29,4 \\ \text { Rivière-Ouelle } & 20 & 15,7 & 15,3 \\ \text { Saint-Nicolas } & 22 & 17,3 & 24,7 \\ & 2 & 1,6 & 2,4\end{array}$

23 Voir à ce sujet, Jacques Lavigne, Mesure des migrations au Canada sous le régime français. Mémoire de maîtrise (démographie) (Université de Montréal, 1974). Les urbains se répartissent ainsi:

$\begin{array}{ccccc}\text { Beaumont } & \text { Charlesbourg } & \text { Neuville } & \text { Rivière-Ouelle } & \text { Saint-Nicolas } \\ 14 & 55 & 19 & 4 & 4\end{array}$

24 Il n'est pas possible à l'échelle régionale de vérifier si des localités sont exclues dans le choix des conjoints ou si les jeunes gens, tentant d'établir un monopole sur les filles à marier, s'efforcent de constituer un isolat. Voir Vu Tien Khang et Sévin, op. cit., 57ss et Burguières, op. cit., 44-45. Par contre rien ne nous a laissé entrevoir cette possibilité. 
TABLEAU II

Provenance régionale des conjoints étrangers du gouvernement de Québec*

\begin{tabular}{|c|c|c|c|}
\hline$t$ & $\begin{array}{c}\text { Charles- } \\
\text { bourg }\end{array}$ & Neuville & $\begin{array}{c}\text { Rivière- } \\
\text { Ouelle }\end{array}$ \\
\hline
\end{tabular}

Charlevoix

Côte de Beaupré

Ile d'Orléans

Environs de Québec

Portneuf

Lotbinière

Lévis

Beauce

Côte du Sud

Kamouraska

-
22
15
1
2
15
-
39
4

$\begin{array}{rr}1 & - \\ 18 & 7 \\ 6 & 4 \\ 102 & 27 \\ 14 & 44 \\ 1 & 10 \\ 1 & 1 \\ - & - \\ 14 & 5 \\ 5 & -\end{array}$

$\begin{array}{rr}- & - \\ 1 & 9 \\ 1 & 4 \\ 6 & 6 \\ - & 5 \\ 1 & 40 \\ - & 11 \\ - & - \\ 7 & 4 \\ 48 & -\end{array}$

*La Beauce n'est ouverte à la colonisation qu'en 1737 et la population totale de Charlevoix n'est que de 227 en 1721 .

En somme, la configuration géographique de la colonie et le mode d'occupation des terres conduisent naturellement à étendre le tissu des relations parentales au-delà des frontières paroissiales. Cet accommodement toutefois n'est pas mieux vu que l'alliance avec une personne ayant vécu jusque-là loin de la paroisse. En Nouvelle-France, la distance n'a pas l'influence déterminante qu'on lui reconnaît en France sur le choix du conjoint. La proximité géographique n'est pas le facteur explicatif de premier rang dans la compréhension des stratégies matrimoniales.

\begin{tabular}{|c|c|c|c|c|c|c|}
\hline \multirow[b]{2}{*}{ Années } & \multicolumn{5}{|c|}{$\begin{array}{l}\text { TABLEAU III } \\
\text { Moyenne décennale des mariages exogames }\end{array}$} & \multirow[b]{2}{*}{$\begin{array}{l}\text { Moyenne } \\
\text { de } \\
\text { l'ensemble* }\end{array}$} \\
\hline & Beaumont & $\begin{array}{c}\text { Charles- } \\
\text { bourg }\end{array}$ & Neuville & $\begin{array}{c}\text { Rivière- } \\
\text { Ouelle }\end{array}$ & $\begin{array}{l}\text { Saint- } \\
\text { Nicolas }\end{array}$ & \\
\hline $1700-1709$ & $73,1 \%$ & $19,2 \%$ & $26,9 \%$ & $15,6 \%$ & $45,5 \%$ & $27,7 \%$ \\
\hline $1710-1719$ & $57,1 \%$ & $34,0 \%$ & $35,3 \%$ & $22,6 \%$ & $43,5 \%$ & $35,9 \%$ \\
\hline $1720-1729$ & $47,3 \%$ & $41,5 \%$ & $55,7 \%$ & $15,0 \%$ & $35,0 \%$ & $42,7 \%$ \\
\hline $1730-1739$ & $45,2 \%$ & $36,7 \%$ & $41,4 \%$ & $50,0 \%$ & $68,0 \%$ & $43,0 \%$ \\
\hline $1740-1749$ & $51,8 \%$ & $34,3 \%$ & $32,8 \%$ & $67,5 \%$ & $63,4 \%$ & $44,5 \%$ \\
\hline $1750-1759$ & $54,8 \%$ & $53,8 \%$ & $45,8 \%$ & $45,9 \%$ & $33,3 \%$ & $48,2 \%$ \\
\hline TOTAL & $52,3 \%$ & $38,5 \%$ & $39,9 \%$ & $39,4 \%$ & $48,2 \%$ & $41,9 \%$ \\
\hline
\end{tabular}




\section{Ventilation de la présence des conjoints étrangers}

Les variations dans le temps et par paroisse de l'importance numérique des étrangers éclairent un peu plus la question. Pour les fins de l'analyse, nous avons retenu quatre périodisations: le rythme long qui ressort de la somme des mariages pendant trois générations, les moyennes décennales, les variations mensuelles et les données annuelles. Chacune illustre un aspect des stratégies matrimoniales. Leur poids respectif permet de dégager les préoccupations majeures et les contextes économiques ou sociaux les plus favorables à la présence de conjoints étrangers au mariage.

Les résultats d'ensemble montrent une importance numérique croissante des conjoints étrangers entre 1700 et 1760 .

D'une décennie à l'autre, la moyenne de 27 à 35 passe à 42 , à 43 , à 44 et finalement à $48 \%$. Si l'on s'en tenait à cette seule variable, on pourrait croire à un relâchement de la cohésion paroissiale après qu'elle eût atteint un sommet au début du XVIIIe siècle. Par la suite, il se produirait dans l'ensemble une ouverture lente mais continue envers l'étranger.

En corollaire, il faut noter une réduction des écarts entre les moyennes paroissiales et la moyenne générale. Tandis que l'écart est de $57 \%$ ( 73 vs 16) dans la première décennie, il n'est plus que de $22 \%$ (55 vs 33 ) dans la dernière. Chaque paroisse tend en somme vers une sorte de conformité en se rapprochant de la moyenne générale. Cette tendance se produit partout, mais pas nécessairement en même temps.

Aussi cet éclairage général reste-t-il insuffisant pour expliquer complètement l'importance et les variations de la présence des conjoints étrangers. En effet, les écarts d'une paroisse à une autre et d'une décennie à une autre sont assez grands pour inciter à chercher dans les conditions locales de développement les éléments d'une explication significative.

Notre analyse des moyennes décennales met en relation les facteurs de population (l'ancienneté du peuplement, le bassin de population et donc de conjoints possibles), d'espace (la proximité ou l'éloignement du centre urbain), de temps (le rythme de croissance dans ces paroisses). La disponibilité de conjoints dans la paroisse est liée à l'ancienneté du peuplement et au bassin de population. L'analyse comparée de l'évolution de la présence de conjoints étrangers révèle des similitudes et des différences particulièrement intéressantes.

Dans les deux paroisses les plus anciennes et les plus peuplées, Charlesbourg et Neuville, la moyenne décennale de conjoints 
étrangers suit une courbe en tous points identique tout au long de la période. De plus, ces paroisses sont celles où, au total, il entre le moins d'étrangers: 2 à $4 \%$ sous la moyenne ${ }^{25}$. Cela tendrait à confirmer l'hypothèse du resserrement de la cohésion sociale et à infirmer celle d'une ouverture à l'étranger. Mais ces données générales sont trompeuses. En fait, ces paroisses vieilles et populeuses sont celles où il entre le moins d'étrangers au début du XVIIIe siècle et, par la suite, elles en admettent de plus en plus ${ }^{26}$. Ainsi, il semble bien qu'il faille écarter l'hypothèse d'une cohésion sociale renforcée, fondée sur une mesure de l'endogamie à l'échelle paroissiale ${ }^{27}$. Ou bien le milieu paroissial s'ouvre graduellement à l'étranger ou bien la cohésion sociale, par le fait que les familles ont essaimé ailleurs, prend d'autres formes et repose sur des facteurs familiaux ou socio-professionnels.

D'un autre côté, dans les deux paroisses les moins peuplées, Saint-Nicolas et Rivière-Ouelle, la présence de conjoints étrangers suit également une courbe similaire, caractérisée par un décollage tardif, vers 1730, et un palier élevé qui se poursuit jusqu'en 1750 . Quant à Beaumont, peu peuplée au début, elle reçoit, tôt au XVIIIe siècle, beaucoup d'étrangers: $70 \%$ des unions sont exogames. Par la suite, elle affiche une stabilité remarquable, se tenant un peu au-dessus de la moyenne générale.

Il semble ressortir de cette analyse deux explications majeures moins paradoxales que complémentaires. D'une part, dans les paroisses numériquement faibles, l'arrivée d'étrangers atteint des niveaux très élevés - supérieurs à $60 \%$ - au moment où la colonisation prend un essor. D'autre part, les paroisses anciennes et populeuses, après avoir atteint un seuil, soit de terres disponibles, soit de conjoints possibles par suite des alliances familiales, soit d'autarcie paroissiale, accueillent de plus en plus d'étrangers. Il s'agit alors de compenser le manque de terres et de préserver le patrimoine, ou bien le manque de conjoints et d'assurer la reproduction sociale. Les vieilles paroisses pourvoient en population les nouvelles zones de colonisation en même temps qu'elles deviennent plus réceptives au mariage avec un étranger.

L'inscription, dans le temps, de ces comportements différenciés en regard du bassin de population, révèle une sorte de cycle. Dans un premier temps, la paroisse fait considérablement appel à subdivisée.

25 Il faut exclure Rivière-Ouelle à cause de son immense étendue avant qu'elle soit

26 Seulement 19 et $20 \%$ au cours de la première décennie en regard de la moyenne générale de $41,9 \%$. Voir Tableau III.

27 La période étudiée joue ici un rôle déterminant. À d'autres époques, l'hypothèse peut être retenue et donner des résultats valables. Louise Dechêne, op. cit., 104. 
l'étranger pour amorcer sa croissance. La génération suivante, pourvue d'un éventail large de résidents, ferme la porte à l'étranger. Les seuils atteints par la suite l'obligent à déverser ses surplus de population et cela entraîne une réouverture graduelle à l'étranger. En schématisant bien sommairement le phénomène, on pourrait croire que la paroisse attire l'étranger grâce aux terres disponibles et, plus tard, l'accepte pour placer ses filles.

Le deuxième facteur, celui de la localisation, ajoute une explication aux variations de l'importance numérique des étrangers. Richard Colebrook Harris, par exemple, explique en partie l'évolution du régime seigneurial par le fait que la ville joue un rôle $\mathrm{d}^{\text {'attrait }}{ }^{28}$. Selon cette hypothèse, plus la seigneurie est située près de la ville, plus son développement est rapide. Par la suite, le peuplement gagne graduellement des régions de plus en plus éloignées.

Cette hypothèse se vérifie en partie dans le gouvernement de Québec. À Rivière-Ouelle, le décollage est tardif à cause de l'éloignement de la paroisse et ne s'effectue qu'au moment où les vides ont été comblés, lorsque la colonisation rejoint ce territoire. Par contre, comment expliquer que Charlesbourg avec 19\%, puis $34 \%$ d'alliances exogames paraisse si peu invitante au début du XVIIIe siècle? Pourquoi la situation de Neuville au début (27\% d'unions comprenant un étranger) est-elle si éloignée de celle de Beaumont qui en compte $73 \%$ ? Mais surtout comment expliquer le retard de Saint-Nicolas?

À n'en pas douter, la localisation de la paroisse pèse beaucoup moins que le bassin de population dans l'explication de la présence d'étrangers. Et, sous cet angle, les conditions locales de développement paraissent jouer un rôle primordial. Mais il n'a pas été possible d'établir des indices de croissance satisfaisants pour conduire à une preuve tangible. Des éléments tels la construction de chemins, le remplacement d'église, le passage de la desserte à la cure fixe et l'ouverture de nouveaux rangs restent des facteurs trop imprécis. Ces indices un peu vagues s'ajoutent aux paradoxes que révèle un rythme de croissance fondé sur le nombre de mariages et de baptêmes.

Ainsi, au moment où la croissance de la population paraît forte à Beaumont (1730-1750) et à Charlesbourg (1740-1750), la proportion de conjoints étrangers diminue considérablement. À d'autres occasions, il y a adéquation entre une croissance faible et le petit nombre d'étrangers (Neuville entre 1740 et 1750 et SaintNicolas entre 1720 et 1730). A l'inverse, croissance forte et apport

28 Richard Colebrook Harris. The Seigneurial System in Early Canada. A Geographical Study (Québec, PUL, 1968), 114-116. 
considérable d'étrangers vont de pair à Rivière-Ouelle entre 1730 et 1760 et à Saint-Nicolas entre 1730 et 1740 (voir annexe II).

La réinsertion dans le temps de ces évolutions différenciées rapproche encore des conclusions relatives au cycle de la présence d'étrangers. La mesure des variations mensuelles apporte peu d'éclairage nouveau aux facteurs d'explication des alliances exogames. En fait, cette analyse démontre qu'à l'instar des résidents, les étrangers se marient principalement durant les mois de novembre et de janvier et s'en abstiennent en décembre et mars.

Étant donné la nature des variations, l'analyse des données annuelles représente la forme de périodisation la plus satisfaisante dans la recherche des explications aux nombreuses alliances exogames. Dans la mesure où l'importance numérique des étrangers suit assez exactement celle du nombre de mariages, elle traduit une compensation à un besoin local. En fait, les variations annuelles du nombre d'alliances exogames permettent de dégager les effets de circonstances ou de conjonctures.

\section{Circonstances de la formation d'unions exogames ${ }^{29}$}

Le nombre d'unions exogames varie suffisamment d'année en année et d'une paroisse à l'autre pour qu'il soit possible de repérer et d'ordonner les éléments qui influencent le plus leur formation. Les lois du régime démographique ancien veulent qu'après une multiplication des décès les courbes des mariages subissent une chute radicale. Les années suivantes, un phénomène de compensation devrait se produire. La courbe des décès diminue pendant que celle des mariages et des naissances augmente. Cette affirmation se vérifie fréquemment dans nos relevés ${ }^{30}$. Aussi, nous avons voulu déterminer jusqu'à quel point de semblables compensations pouvaient entraîner une augmentation des alliances exogames.

Les facteurs favorables à de telles unions peuvent être accidentels, comme une épidémie, présenter un caractère général mais occasionnel, comme le veuvage, ou encore relever de circonstances exceptionnelles, comme la guerre. Ces distinctions ont permis de formuler trois hypothèses. Si, après de fortes mortalités, la courbe des mariages augmente sensiblement et que celle des unions exogames subit la même poussée, nous pourrions avancer l'idée que lorsque la mortalité adulte est excessive, les étrangers sont plus facilement acceptés du fait qu'ils comblent un vide. Si la présence

II.

29 Toutes les données utilisées dans cette section sont tirées des tableaux de l'annexe

30 C'est le cas à Rivière-Ouelle entre 1698 et 1702 , à Charlesbourg en 1711 et 1714 , etc. Voir annexe II. 
d'étrangers est relativement stable mais se trouve plus concentrée dans les remariages, cela pourrait signifier des pratiques sociales différentes selon l'état civil du conjoint résident. Si les étrangers sont plus nombreux en période difficile, quand les conditions de vie sont perturbées, nous pourrions en déduire que, dans ce cas, le relâchement du tissu social est assez important pour inciter à la tolérance.

La vérification de ces hypothèses est complexe car les données de base varient dans le temps, d'une paroisse à une autre et d'une situation à une autre. Par exemple, les données du veuvage et du remariage diffèrent de celles des épidémies en même temps qu'elles se recoupent. Il n'en est pas moins important de tenter de vérifier laquelle de ces circonstances favorise le plus l'alliance avec un étranger. À la limite, cela peut indiquer un comportement envers ces inconnus et les difficultés ou les facilités qui leur sont faites.

En ce qui concerne les épidémies, il n'est pas suffisant de se fier aux résultats globaux pour en mesurer l'impact réel. Prise dans son ensemble, 1703 est une année record pour les sépultures, excepté quelques cas en fin de période. Dans le détail cependant deux des cinq paroisses ne sont pas touchées, ce qui renforce l'ampleur des décès dans les trois autres. Les épidémies peuvent aussi se propager plus ou moins vite dans diverses régions géographiques. En 1711, les paroisses de Charlesbourg et Neuville sont victimes d'une épidémie de variole qui n'atteindra Saint-Nicolas qu'en 1712.

Une autre nuance doit être ajoutée: elle concerne les personnes touchées par la maladie. En 1711, à Charlesbourg, l'épidémie

TABLEAU IV

Lieux et années de fortes mortalités suivies d'un nombre élevé d'alliances exogames.

$\begin{array}{ll}1711 & \text { Charlesbourg } \\ 1712 & \text { Neuville, Rivière-Ouelle, Saint-Nicolas } \\ 1714-1715 & \text { Charlesbourg } \\ 1728 & \text { Charlesbourg } \\ 1731 & \text { Charlesbourg, Neuville, Saint-Nicolas } \\ 1735 & \text { Charlesbourg } \\ 1736 & \text { Neuville } \\ 1742 & \text { Saint-Nicolas } \\ 1743-1744 & \text { Beaumont, Charlesbourg } \\ 1749 & \text { Beaumont, Neuville, Saint-Nicolas } \\ 1755-1756 & \text { Neuville } \\ 1756 & \text { Charlesbourg } \\ 1756-1757 & \text { Rivière-Ouelle }\end{array}$


est plus dévastatrice chez les adultes que chez les enfants ( 37 vs 26), tandis que l'inverse se produit à Neuville (6 adultes, 23 enfants et bébés). Par contre en 1733, dans quatre paroisses, les enfants/bébés décèdent en très grand nombre et les adultes sont épargnés. Parfois la maladie frappe à plusieurs endroits, simultanément ou non, concerne ou pas les mêmes tranches d'âge, tout comme elle peut être propre à un endroit et à une catégorie de personnes. Chaque fois, la courbe des mariages est affectée différemment. C'est pourquoi, au-delà d'une vision d'ensemble, il est nécessaire de connaître les vicissitudes de chaque paroisse.

En général, après une épidémie touchant plusieurs adultes, le nombre de mariages exogames augmente de façon notable. Cette mortalité favorise par la suite la venue d'étrangers. Par contre, la mort de nombreux adultes n'est pas toujours suivie de l'entrée d'étrangers. À Rivière-Ouelle en 1701, dix mariages sont célébrés, mais un seul étranger est concerné et aucun veuf. On fait sensiblement la même observation à Charlesbourg et Neuville en 1703, à Rivière-Ouelle en 1711-1712, et à Saint-Nicolas en 1754. Au total, malgré quelques contre-exemples, il est manifeste que les épidémies favorisent la venue d'étrangers.

La mise en relation des épidémies et du veuvage ainsi que l'analyse de leur impact respectif sur l'arrivée d'étrangers éclairent davantage les pratiques sociales. Après les épidémies de 1703, 1711,1712 et 1715 , soit à Charlesbourg, soit à Neuville, les mariages de veufs sont plus nombreux que les mariages avec un étranger. Après 1716 , le nombre d'étrangers dépasse régulièrement celui des veufs qui se remarient. Cette tendance confirme le repliement observé dans les anciennes paroisses par l'analyse des moyennes décennales, repliement suivi de l'ouverture progressive aux étrangers.

Le veuvage n'en est pas moins une forme d'accident susceptible de favoriser l'arrivée d'étrangers. Par son caractère constant et répétitif, il aurait pu jouer un rôle privilégié dans la formation d'unions exogames ${ }^{31}$. La possibilité pour les veuves de se remarier, les raisons pour lesquelles elles sont recherchées, l'origine et l'état civil de leurs prétendants sont autant de questions que nous nous sommes posées.

31 Nous ignorons toutefois quelle proportion de veufs et de veuves se remarient et nous les rejoignons inégalement. Nous n'avons pu retracer les veufs qui ont pris épouse à l'extérieur. Par contre, on peut croire que le relevé comprend la majorité des veuves qui se remarient, à cause des prescriptions de l'Église. La recherche vaut donc essentiellement pour les veuves. Quelques chercheurs ont approfondi cette question dans une perspective différente de la nôtre. Charbonneau, op. cit., et Dechêne, op. cit., 107. 
Pour préciser l'influence de cette situation, la relation remariages/étrangers a été mesurée de diverses façons. Dans quatre des cinq paroisses, le nombre d'étrangers n'est que de 5 à $10 \%$ plus élevé dans les remariages que dans les premières alliances, signe de la facilité pour une veuve de contracter une nouvelle union avec un célibataire résident. D'un autre côté, le remariage des veuves en Nouvelle-France a fourni des données qui diffèrent sensiblement des études françaises. Dans le canton du Bleymard, les mariages entre un célibataire et une veuve sont beaucoup plus rares que les remariages entre un veuf et une célibataire ${ }^{32}$. Dans les paroisses étudiées, la proportion de ces remariages semble mieux équilibrée; si elle n'est que de $24 \%$ à Beaumont et $30 \%$ à Neuville, elle atteint 42,43 et $46 \%$ à Charlesbourg, Rivière-Ouelle et Saint-Nicolas.

Être veuve en Nouvelle-France n'est pas une disgrâce car le remariage ne semble pas soumis aux mêmes restrictions qu'en France où, écrit Edward Shorter, les remariages avec des jeunes célibataires étaient réprouvés et soumis à charivari ${ }^{33}$. Pour Pierre Goubert, la veuve de 35 à 45 ans, dans le Beauvaisis, trouve difficilement à se remarier ${ }^{34}$. Dans la colonie, le statut de veuve ne semble pas un handicap; celle-ci n'est cantonnée ni aux veufs, ni aux étrangers. Bien que les veuves ne semblent pas pénalisées dans la recherche d'un époux, certaines rencontrent des difficultés. En fait, on peut présumer que les raisons pour lesquelles certaines veuves contractent un mariage exogame sont plus complexes. Les questions d'âge, de charge de famille et d'attirance restent à préciser. Toutefois, si les résidents font défaut, elles peuvent tenter leur chance, avec ou sans aide, du côté des étrangers.

C'est ainsi qu'à Beaumont les veuves les accueillent sur leur exploitation, tandis que les veufs de la paroisse vont facilement chercher femme en dehors. À Neuville, elles bénéficient certainement de la connivence du curé qui, par son accord, les fait bénéficier d'une tolérance sociale plus grande et les incite carrément à épouser un mari venant parfois de fort loin. Elles profitent de l'aide indirecte de l'État à Charlesbourg, où ce n'est pas par hasard que les célibataires qui marient une veuve sont si souvent originaires de France et soldats.

Dans ces deux derniers cas, l'Église et l'État, de par leurs actions, interviennent pour amener les familles privées de chef à retrouver un appui. A Charlesbourg, le nombre proportionnellement élevé des soldats épousant des veuves ne peut s'expliquer uni$27,3 \%$ ).

$32 \mathrm{Vu}$ Tien Khang et Sévin, op. cit., 36. (35 veuves en regard de 93 veufs soit

33 Edward Shorter, Naissance de la famille moderne (Paris, Seuil, 1977), 271.

34 Pierre Goubert, Cent mille provinciaux au XVIIe siècle (Paris, Flammarion), 59. 
quement par la recherche des terres. Ce choix est probablement influencé par une pratique administrative d'hébergement des soldats chez les veuves. En somme, pour pallier aux difficultés des familles, l'administration leur fournit indirectement un soutien, espérant que ce palliatif deviendra permanent.

L'exemple d'un curé se faisant entremetteur et favorisant des alliances avec des gens de l'extérieur se retrouve à Neuville entre 1748 et 1752 . Jusqu'à cette date, on comptait en moyenne un remariage par an; or pendant ces quatre ans, le curé LouisEustache Chartier de Lotbinière célèbre 16 remariages. Durant le même laps de temps, huit orphelins de père et mère trouvent un conjoint tandis que la moyenne antérieure et postérieure est d'un cas par an. Il semble évident que ce curé cherche à venir en aide aux familles en difficulté et n'hésite pas à faire venir de très loin, bien qu'en provenance de la colonie, des conjoints pour ses protégés.

Enfin, les variations du nombre des mariages exogames semblent plus marquées lors de périodes difficiles. Sans vouloir accorder une importance démesurée aux faits militaires, nos observations nous poussent à établir des corrélations entre le climat difficile des années troublées et le nombre d'étrangers. À SaintNicolas, Charlesbourg et Rivière-Ouelle, leur proportion au mariage est très forte à la veille et à la suite de la guerre de Succession d'Autriche (80\% en $1742-1743$ et $75 \%$ en $1749-1750)$. À Rivière-Ouelle pendant la décennie 1740-1749, c'est la présence de Français qui donne à la paroisse son taux record d'étrangers. À Charlesbourg, le nombre de soldats qui épousent des veuves est assez élevé: trois en 1747, huit des dix étrangers en 1757, trois en 1758 , deux en $1759^{35}$. La cohésion paroissiale semble davantage menacée lors des conflits.

Notre tentative d'explication des alliances exogames a permis de mettre en évidence des particularismes locaux qui ont des répercussions occasionnelles, mais sensibles et mesurables. Les événements liés à la conjoncture, comme l'épidémie, l'hébergement des soldats ou l'intervention d'un curé, exercent finalement une plus grande influence sur l'entrée d'étrangers qu'une situation comme le veuvage. La facilité qu'ont les veuves de se remarier fait que leur situation ne nécessite pas une tolérance particulière bien évidente,

35 Ces soldats sont identifiés par leur profession dans l'acte de mariage. Une vérification plus poussée permettrait certainement de hausser ces chiffres, si l'on se fie à l'utilisation des surnoms, à l'état d'orphelin et aux témoins au mariage.

Quant aux Français qui se marient à Rivière-Ouelle, il s'agit vraisemblablement d'une partie d'hommes de recrue incorporés dans les troupes et de faux-sauniers répartis chez des habitants, que l'intendant Hocquart fit venir. AC, Beauharnois et Hocquart à Maurepas, 2 oct. 1739, C 11A, vol. 71: f. 9-10. 
mais parfois une intervention extérieure exceptionnelle. Chacun de ces cas révèle des situations difficiles à vivre, auxquelles on apporte une solution ponctuelle.

\section{Colonisation et parenté}

La présence de conjoints étrangers au mariage peut s'expliquer par d'autres facteurs. Les progrès de la colonisation dans une paroisse peuvent inciter les futurs époux à venir y chercher en même temps femme et pays. L'existence de liens de parenté entre les futurs mariés peut aussi faciliter la venue d'étrangers dans une paroisse ancienne ou leur regroupement dans des paroisses nouvelles. Le cas de Beaumont, qui connaît ces deux stades de développement au cours de la période étudiée, permet de donner des éléments de réponse.

Au tournant du siècle, la seigneurie de Beaumont, concédée en 1672 , vient à peine de s'ouvrir à la colonisation. Le seigneur Charles-Thomas Couillard a distribué les 31 censives du premier rang entre 1680 et 1699 , dont 29 à des étrangers. La majorité, soit près de $75 \%$ s'établit à Beaumont en permanence; un seul concessionnaire va marier une fille de la paroisse quelque temps après avoir obtenu sa terre. Maris autant que femmes sont donc étrangers au milieu. Lors de la distribution des 12 censives du troisième rang, en 1707, seulement quatre étrangers ont droit à des terres; ils étaient tous mariés, et deux l'étaient avec des filles de la paroisse. Lorsque Charles-Marie Couillard concède 17 nouvelles censives au deuxième rang en 1723, il n'en accorde que quatre à des étrangers dont deux à des cousins, qui ont déjà pris femme à Beaumont. Par son pouvoir de concéder, le seigneur assure une certaine cohésion sociale, en limitant l'entrée d'étrangers lorsque les gens en place sont assez nombreux pour assurer la relève. Aussi l'arrivée de conjoints étrangers ne peut être attribuable à la possibilité pour eux d'obtenir une terre.

Toutefois, des 112 étrangers qui se marient à Beaumont entre 1700 et 1760, 30 s'y établissent. Certains se marient, résident chez des parents, puis obtiennent une terre quelques années plus tard. D'autres avaient déjà acquis une censive avant leur mariage et décident de faire souche à Beaumont. Quelques-uns épousent des veuves déjà pourvues d'un bien foncier. L'établissement de conjoints étrangers à Beaumont atteint son plus haut sommet durant les années 1738-1746, années correspondant d'ailleurs à un fort pourcentage d'unions exogames. Cette période coïncide aussi avec une forte poussée de colonisation aux troisième et quatrième rangs. Ainsi, tout comme pour les années de mise en place, les con- 
joints étrangers viennent en grand nombre et sont facilement acceptés. Il y a donc concordance entre leur présence et les progrès de la colonisation. Toutefois, étant donné que les nouvelles concessions sont attribuées aux résidents et à leurs enfants, il est impossible de percevoir le mariage comme un moyen d'appropriation des biens fonciers. La saturation du milieu et la nécessité d'avoir recours à des étrangers pour se regénérer expliquent mieux leur présence.

Les liens de parenté entre des résidents et des conjoints étrangers jouent également un rôle significatif. Des 112 étrangers venus se marier à Beaumont, 50 avaient des liens de parenté proches avec des gens de la paroisse ${ }^{36}$; ce nombre élevé accentue le caractère endogame du mariage et élimine du même coup la part du hasard dans les alliances exogames, tout en témoignant d'une forte cohésion sociale.

Même si cette proportion de parents n'a pas été calculée pour toutes nos paroisses, quelques exemples démontrent l'importance de cette pratique sociale. Ainsi, à Saint-Nicolas, sur huit conjoints qui viennent de Château-Richer, une paroisse éloignée, six appartiennent à la famille Gagnon, dont quatre épousent des gens de Saint-Nicolas appartenant aux familles Dubois et Demers. Il existe une relation semblable entre les familles Dupéré de Charlesbourg et Fréchet de Saint-Nicolas, de même qu'entre les Simonaud de Pointe-à-la-Caille et les Lambert de Saint-Nicolas. À RivièreOuelle, parmi les 24 étrangers provenant des autres régions, neuf avaient des liens de parenté avec des résidents de la paroisse. La recherche des liens familiaux entre les étrangers et la communauté renforce aussi l'image d'une société où les liens sont très étroits. L'analyse patronymique confirme également l'importance des liens familiaux dans les alliances exogames. À Neuville, par exemple, 47 des 130 conjoints étrangers $(35,3 \%)$ ont un patronyme déjà identifié dans la paroisse.

Nous sommes bien conscients que les comportements relatifs à la consanguinité ont des dimensions religieuses et sociales parfois déterminantes. En Nouvelle-France, la faiblesse numérique de la population et sa dispersion sur un vaste territoire se sont traduites par une très faible densité de l'occupation humaine, ce facteur étant susceptible d'intensifier la consanguinité. À l'inverse, toutefois, et peut-être en conséquence, le discours de l'Église contre les mariages consanguins est très fort et s'appuie sur un tarif de dispenses très élevé:

36 Cette analyse repose sur des fiches de famille élaborées à partir des registres d'état civil et complétées à l'aide du dictionnaire généalogique de Cyprien Tanguay. 


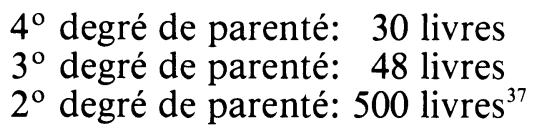

et il est devenu impressionnant au point de passer dans la culture et l'éducation populaire. D'un autre côté, l'historien et anthropologue français Pierre Lamaison a bien démontré que, dans un monde clos, les gens prenaient tout de même grand soin de ne pas se marier entre parents: le mariage consanguin apparaissant incompatible avec la logique même du système d'échange et de transmission des biens patrimoniaux.

Dans les paroisses étudiées, les dispenses de consanguinité sont en nombre extrêmement faible. Elles représentent 9,5\% du total des dispenses et concernent $2,2 \%$ des mariages recensés. Une répartition chronologique atténue encore davantage l'importance des mariages consanguins. La première dispense accordée l'est en 1728. Par la suite, la moitié de ces dispenses (17) sont concentrées pendant des périodes de guerre, soit 5 en 1747-1748 et 12 entre 1756-1758.

Par ailleurs, la taille des paroisses joue à l'encontre de ce type de mariages. Dans la paroisse la plus populeuse, Charlesbourg, nous ne comptons que deux dispenses pour consanguinité. À l'inverse, la petite paroisse de Saint-Nicolas en compte dix. Cela accentue encore plus l'impression que les gens évitent, dans la mesure du possible, d'épouser un parent, mais la rareté de conjoints disponibles les y pousse parfois. Dans 50\% de ces alliances, le consanguin est un étranger à la paroisse, ce qui semble confirmer cette nécessité de recourir parfois à un parent résidant à l'extérieur pour consolider une famille en difficulté. Ce qui confirme aussi que la famille joue un rôle significatif dans la venue d'étrangers.

En compilant ces données sur les dispenses de consanguinité, nous avons noté que les dispenses, en général, fournissaient des éléments d'analyse intéressants pour étudier les comportements qu'elles sous-tendent et en particulier face à la venue d'étrangers. Pour nos cinq paroisses nous avons dénombré 356 dispenses mais, comme pour les autres inscriptions, cette donnée souffre probablement d'un sous-enregistrement constant, impossible à rectifier. Cela constitue tout de même une base d'analyse valable. La dispense, surtout celle donnée à un étranger ou à un consanguin, n'est pas un geste gratuit ou formel. Le curé prend des précautions; il est attentif à ne pas se faire jouer par des gens peu connus: soldats, navigateurs ou immigrés de fraîche date, qui oseraient tenter de 400 .

37 P.-A. Leclerc, «Le mariage sous le régime français», RHAF, XIII, 3 (déc. 1959): 
déjouer les règles de l'Église. Il n'est pas rare qu'un étranger doive présenter une lettre d'un prêtre qui le connaît ou de son capitaine au moment de publier les bans.

Le regroupement des individus ou des couples qui ont demandé des dispenses de bans a révélé que près de $40 \%$ sont accordées à des étrangers, $23,8 \%$ à des veufs ou veuves, $7 \%$ aux membres des élites locales, $3,1 \%$ à cause d'alliances spirituelles, $2 \%$ aux conjoints ayant déjà eu des enfants hors mariage, 9,5\% dans des cas de consanguinité et $16,3 \%$ pour des raisons qui ne sont pas précisées. Il va de soi que les raisons invoquées ou implicites pour demander une dispense sont parfois complexes. Par exemple, il peut s'agir d'un conjoint qui est à la fois veuf, étranger et parent. Au total, les doubles ou triples comptes ne gonflent le nombre des dispenses que de $29 \%$. Par ailleurs, sauf quand il est nécessaire de se conformer aux règles de l'Église - consanguinité et alliance spirituelle - les dispenses traduisent une certaine forme de pudeur: voulue et bien compréhensible dans le cas de veufs ou veuves et de parents d'enfants illégitimes, un peu trop affichée de la part des membres de l'élite locale. Rien ne laisse croire qu'un sentiment différent de celui-là ait présidé à la demande de dispenses lors du choix d'un étranger comme conjoint. Le nombre de ces demandes est trop élevé pour être l'effet du hasard. Manifestement, l'on tient à réduire la publicité accordée à ces alliances, voire peut-être - et c'est une supposition - à ces mésalliances pour la paroisse. Il serait certes un peu forcé, sans preuves complémentaires, de voir dans cette hypothèse l'indice majeur des réticences à rompre la cohésion paroissiale. Il n'en reste pas moins que dans un cas sur cinq la présence d'un étranger incite à demander une dispense: reflet certain d'une perception qu'il reste à mieux cerner.

L'analyse des alliances matrimoniales nous a fait découvrir un personnage: l'étranger, un étranger dont l'importance numérique est si considérable - $42 \%$ dont $58 \%$ viennent de loin - qu'elle renverse certains préjugés sur la cohésion paroissiale.

Prenant pour acquis que l'affinité ne s'improvise pas, qu'on épouse sinon un familier du moins une connaissance, nous avons cherché à identifier les facteurs qui entrent en ligne de compte pour expliquer sa présence. Nous avons finalement dressé de cet étranger un portrait circonstanciel qu'il reste à compléter.

De fait, cette analyse fournit surtout l'esquisse d'un personnage et un aperçu des motifs de sa présence. Ce personnage, géné- 
ralement un homme du monde rural, est présent dans deux mariages sur cinq. Souvent il réside à proximité de son futur conjoint, mais habituellement il vient d'assez loin. Les motifs de sa venue peuvent être différents de ceux de son intégration au milieu. Mais il semble davantage accepté pour pallier à des situations difficiles, et sa présence au mariage n'est pas nécessairement suivie d'un établissement permanent. La compensation à un besoin fortement ressenti au plan local semble être une règle guidant les comportements de tolérance et d'acceptation à son égard.

Si certains étrangers sont attirés par les terres disponibles, en particulier par celles qui sont défrichées, cet avantage leur est procuré surtout parce qu'ils remédient à des situations exceptionnelles, notamment pour combler les vides causés par les épidémies. Le veuvage, quant à lui, n'a que très peu d'effets sur la constitution d'alliances exogames. La situation paraît avoir été si bien intégrée socialement que la veuve éprouve rarement de difficulté à se remarier. L'hébergement de soldats ou l'entremise d'un curé semblent avoir encore plus d'influence que la situation même. Il faut noter que le discours implicite ou explicite de l'Église et de l'État en cette matière a pu jouer un rôle important dans l'instauration de pratiques sociales de tolérance.

À une autre échelle, l'ancienneté du peuplement et le bassin de population sont des facteurs plus déterminants que le rythme de développement ou la localisation de la paroisse pour expliquer la présence de conjoints étrangers. Les larges horizons de l'espace québécois ont réduit sensiblement l'importance de la proximité géographique.

Finalement, la famille et le patrimoine à préserver semblent au premier rang des stratégies matrimoniales. La multiplicité des doubles alliances et des renchaînements d'alliances ainsi que le nombre d'étrangers ayant des parents dans la paroisse où ils se marient, sont des indices valables. Ainsi l'étranger est appelé à jouer deux rôles: il peut prendre temporairement le relais dans une famille en difficulté, ou être utile pour placer les enfants qui n'héritent pas du patrimoine familial.

Transposée au plan paroissial, cette analyse contredit l'image d'une paroisse rurale fermée, réfractaire à l'étranger. Elle oblige également à nuancer fortement l'hypothèse d'une amorce de forte cohésion paroissiale qui se manifesterait par une diminution du nombre de conjoints étrangers, autrement dit par une endogamie plus élevée. Dans le gouvernement de Québec, au XVIIIe siècle, la situation est bien différente. Le nombre de conjoints étrangers est élevé et en augmentation. De plus, c'est justement par cette impor- 
tance considérable et croissante que se révèle une plus grande cohésion, cohésion toutefois qui est davantage familiale et sociale que paroissiale.

Il ne faut pas oublier cependant que cet étranger est réticent à s'afficher, comme le trahit son recours fréquent aux dispenses. Il ne faut donc pas s'étonner que son portrait soit long et difficile à tracer. Seulement au plan circonstanciel, il vaudrait la peine d'évaluer le rôle dynamique de la mort dans la formation de couples et l'intervention de la famille pour combler les lacunes. Encore ce portrait resterait-il à compléter aux plans individuel, familial et social. Ce personnage, si présent dans notre littérature, notre folklore et notre histoire, mérite, à n'en pas douter, une fiche d'identification plus élaborée.

ANNEXE I

ORIGINE GÉOGRAPHIQUE DES CONJOINTS ÉTRANGERS

\begin{tabular}{|c|c|c|c|c|c|c|c|c|c|c|c|c|}
\hline \multirow[b]{3}{*}{ Beaumont } & \multirow{2}{*}{\multicolumn{2}{|c|}{$\begin{array}{l}\text { Paroisses } \\
\text { limitrophes } \\
\text { Nbre } \%\end{array}$}} & \multirow{2}{*}{\multicolumn{2}{|c|}{$\begin{array}{l}\text { Même région } \\
\mathbf{N} \%\end{array}$}} & \multirow{2}{*}{\multicolumn{2}{|c|}{$\begin{array}{c}\text { Autres régions } \\
\mathbf{N} \quad \%\end{array}$}} & \multirow{2}{*}{\multicolumn{2}{|c|}{$\begin{array}{c}\text { Autres } \\
\text { gouvernements } \\
\mathbf{N} \quad \%\end{array}$}} & \multirow{2}{*}{\multicolumn{2}{|c|}{$\begin{array}{c}\text { Extérieur } \\
\text { de la colonie } \\
\mathrm{N} \quad \%\end{array}$}} & \multicolumn{2}{|c|}{ Total } \\
\hline & & & & & & & & & & & & $\%$ \\
\hline & 39 & 34,8 & 39 & 34,8 & 61 & 54,5 & 2 & 1,8 & 10 & 8,9 & 112 & 65,2 \\
\hline Charlesbourg & 104 & 41,9 & 104 & 41,9 & 59 & 23,8 & 7 & 2,8 & 78 & 31,5 & 248 & 58,1 \\
\hline Neuville & 38 & 29,0 & 44 & 33,6 & 54 & 41,2 & 10 & 7,6 & 23 & 17,6 & 131 & 66,4 \\
\hline Rivière-Ouelle & 42 & 47,2 & 48 & 53,9 & 16 & 18,0 & 2 & 2,2 & 23 & 25,8 & 89 & 46,0 \\
\hline Saint-Nicolas* & 38 & 44,7 & 40 & 47,1 & 39 & 45,9 & 1 & 1,2 & 2 & 2,4 & 85 & 49,5 \\
\hline
\end{tabular}


ANNEXE II

PRINCIPALES DONNÉES TIRÉES DES REGISTRES PAROISSIAUX *

* B: Baptêmes MA: Mortalité adulte TM: Total des mariages ME: Mariages avec un étranger MV: Mariages de veufs ou veuves

BEAUMONT

\begin{tabular}{|c|c|c|c|c|c|c|c|c|c|c|c|}
\hline ANNÉES & B & MA & TM & ME & MV & ANNÉES & B & MA & TM & ME & MV \\
\hline 1700 & 10 & - & 1 & 1 & 1 & 1730 & 27 & 1 & 4 & 3 & - \\
\hline 1701 & 11 & - & 1 & 1 & - & 1731 & 30 & 2 & 3 & - & 1 \\
\hline 1702 & 11 & 1 & - & - & - & 1732 & 24 & 2 & 5 & 3 & 1 \\
\hline 1703 & 7 & 2 & 2 & - & 1 & 1733 & 33 & 4 & 6 & 5 & 1 \\
\hline 1704 & 7 & - & 2 & 2 & 2 & 1734 & 32 & 3 & 7 & 1 & - \\
\hline 1705 & 7 & 1 & 2 & $\overline{1}$ & - & 1735 & 32 & 2 & 2 & 1 & 1 \\
\hline 1706 & 12 & - & 1 & 1 & 1 & 1736 & 25 & 3 & 3 & 1 & - \\
\hline 1707 & 12 & - & - & - & - & 1737 & 33 & 2 & 3 & - & 2 \\
\hline 1708 & 9 & 2 & 3 & 2 & - & 1738 & 29 & 4 & 3 & 2 & - \\
\hline 1709 & 10 & 2 & 3 & 3 & - & 1739 & 31 & - & 6 & 3 & - \\
\hline $1700-09$ & 96 & 8 & 15 & 11 & 5 & $1730-39$ & 296 & 23 & 42 & 19 & 6 \\
\hline 1710 & 8 & 2 & 2 & 1 & - & 1740 & 36 & - & 4 & 3 & - \\
\hline 1711 & 19 & 2 & - & - & - & 1741 & 24 & 3 & 5 & 4 & - \\
\hline 1712 & 9 & 1 & 3 & 2 & - & 1742 & 36 & 3 & 9 & 5 & 1 \\
\hline 1713 & 13 & 1 & 1 & - & - & 1743 & 28 & 5 & 3 & 3 & - \\
\hline 1714 & 17 & 1 & 4 & 3 & 1 & 1744 & 36 & 5 & 6 & 5 & 1 \\
\hline 1715 & 12 & - & 1 & 1 & - & 1745 & 37 & 4 & 6 & 2 & - \\
\hline 1716 & 13 & 2 & 3 & 1 & - & 1746 & 54 & 1 & 7 & 1 & 2 \\
\hline 1717 & 15 & 3 & 2 & 1 & - & 1747 & 41 & 4 & 3 & - & - \\
\hline 1718 & 18 & - & 4 & 2 & 2 & 1748 & 50 & 3 & 8 & 2 & 2 \\
\hline 1719 & 13 & 1 & 1 & 1 & - & 1749 & 44 & 8 & 5 & 4 & 1 \\
\hline 1710-19 & 137 & 13 & 21 & 12 & 3 & $1740-49$ & 386 & 36 & 56 & 29 & 7 \\
\hline 1720 & 14 & 1 & 1 & - & - & 1750 & 13 & 6 & 5 & 1 & - \\
\hline 1721 & 9 & 2 & 5 & 4 & 1 & 1751 & 21 & 3 & 6 & 4 & 1 \\
\hline 1722 & 12 & 1 & 3 & 2 & - & 1752 & 15 & 1 & 3 & 3 & - \\
\hline 1723 & 13 & 2 & 7 & 3 & - & 1753 & 19 & 2 & 5 & 2 & - \\
\hline 1724 & 19 & - & 4 & 2 & 2 & 1754 & 20 & 1 & 5 & 3 & 2 \\
\hline 1725 & 16 & 2 & 2 & - & 1 & 1755 & 15 & 2 & 2 & 2 & - \\
\hline 1726 & 21 & - & 3 & 1 & - & 1756 & 18 & 7 & 8 & 4 & 3 \\
\hline 1727 & 6 & 1 & 6 & 3 & - & 1757 & 8 & 1 & 2 & 1 & - \\
\hline 1728 & 21 & 2 & 5 & 2 & - & 1758 & 12 & 5 & 4 & 1 & 1 \\
\hline 1729 & 27 & 4 & 2 & 1 & - & 1759 & 2 & 7 & 2 & 1 & - \\
\hline $1720-29$ & 158 & 15 & 38 & 18 & 4 & $1750-59$ & 149 & 35 & 42 & 22 & 7 \\
\hline
\end{tabular}


CHARLESBOURG

\begin{tabular}{|c|c|c|c|c|c|c|c|c|c|c|c|}
\hline ANNÉES & B & MA & TM & ME & MV & ANNÉES & B & MA & $\mathbf{T M}$ & ME & MV \\
\hline 1700 & 31 & 4 & 2 & 1 & 0 & 1730 & 56 & 7 & 16 & 9 & 3 \\
\hline 1701 & 29 & 0 & 1 & 0 & 1 & 1731 & 62 & 11 & 10 & 4 & 0 \\
\hline 1702 & 43 & 6 & 5 & 2 & 0 & 1732 & 59 & 3 & 15 & 7 & 2 \\
\hline 1703 & 33 & 23 & 10 & 3 & 6 & 1733 & 51 & 8 & 7 & 2 & 1 \\
\hline 1704 & 57 & 2 & 8 & 0 & 4 & 1734 & 63 & 7 & 13 & 0 & 3 \\
\hline 1705 & 44 & 3 & 5 & 0 & 1 & 1735 & 54 & 11 & 12 & 4 & 3 \\
\hline 1706 & 59 & 2 & 8 & 2 & 3 & 1736 & 64 & 4 & 14 & 7 & 5 \\
\hline 1707 & 48 & 1 & 3 & 0 & 0 & 1737 & 63 & 6 & 11 & 3 & 2 \\
\hline 1708 & 52 & 7 & 6 & 2 & 1 & 1738 & 38 & 2 & 7 & 4 & 1 \\
\hline 1709 & 46 & 5 & 4 & 0 & 2 & 1739 & 57 & 5 & 15 & 4 & 1 \\
\hline 1700-09 & 442 & 53 & 94 & 10 & 18 & $1730-39$ & 567 & 64 & 120 & 44 & 21 \\
\hline 1710 & 59 & 4 & 8 & 4 & 2 & 1740 & 77 & 5 & 13 & 3 & 2 \\
\hline 1711 & 38 & 37 & 5 & 2 & 1 & 1741 & 77 & 6 & 12 & 4 & 1 \\
\hline 1712 & 57 & 2 & 26 & 7 & 11 & 1742 & 75 & 8 & 13 & 5 & 4 \\
\hline 1713 & 54 & 1 & 2 & 1 & 1 & 1743 & 68 & 16 & 11 & 2 & 2 \\
\hline 1714 & 61 & 5 & 3 & 1 & 1 & 1744 & 78 & 20 & 7 & 3 & 1 \\
\hline 1715 & 52 & 19 & 12 & 4 & 5 & 1745 & 61 & 7 & 6 & 3 & 2 \\
\hline 1716 & 52 & 0 & 10 & 3 & 3 & 1746 & 80 & 13 & 17 & 7 & 5 \\
\hline 1717 & 40 & 2 & 5 & 1 & 2 & 1747 & 72 & 16 & 25 & 6 & 3 \\
\hline 1718 & 43 & 1 & 9 & 3 & 3 & 1748 & 87 & 10 & 15 & 10 & 3 \\
\hline 1719 & 45 & 1 & 14 & 6 & 4 & 1749 & 78 & 12 & 18 & 4 & 5 \\
\hline 1710-19 & 501 & 72 & 82 & 32 & 33 & $1740-49$ & 753 & 113 & 137 & 47 & 28 \\
\hline 1720 & 51 & 1 & 8 & 4 & 1 & 1750 & 59 & 5 & 17 & 10 & 3 \\
\hline 1721 & 48 & 4 & 3 & 2 & 1 & 1751 & 81 & 14 & 23 & 6 & 6 \\
\hline 1722 & 44 & 5 & 11 & 5 & 0 & 1752 & 87 & 11 & 15 & 8 & 5 \\
\hline 1723 & 55 & 4 & 7 & 3 & 1 & 1753 & 72 & 12 & 17 & 7 & 2 \\
\hline 1724 & 41 & 6 & 4 & 1 & 1 & 1754 & 75 & 14 & 13 & 9 & 2 \\
\hline 1725 & 45 & 5 & 10 & 3 & 1 & 1755 & 78 & 11 & 6 & 0 & 3 \\
\hline 1726 & 51 & 6 & 7 & 2 & 2 & 1756 & 85 & 21 & 11 & 5 & 2 \\
\hline 1727 & 59 & 3 & 8 & 4 & 2 & 1757 & 57 & 13 & 14 & 12 & 4 \\
\hline 1728 & 40 & 11 & 10 & 4 & 0 & 1758 & 60 & 18 & 8 & 4 & 2 \\
\hline 1729 & 62 & 3 & 14 & 6 & 1 & 1759 & 78 & 34 & 16 & 9 & 3 \\
\hline $1720-29$ & 496 & 48 & 120 & 34 & 10 & $1750-59$ & 732 & 153 & 130 & 70 & 32 \\
\hline
\end{tabular}


NEUVILLE

\begin{tabular}{|c|c|c|c|c|c|c|c|c|c|c|c|}
\hline ANNÉES & B & MA & $\mathbf{T M}$ & ME & MV & ANNÉES & B & MA & $\mathbf{T M}$ & ME & $\mathbf{M V}$ \\
\hline 1700 & 36 & 11 & 8 & 0 & 0 & 1730 & 33 & 2 & 6 & 5 & 0 \\
\hline 1701 & 31 & 4 & 2 & 0 & 0 & 1731 & 44 & 10 & 4 & 1 & 0 \\
\hline 1702 & 39 & 14 & 6 & 1 & 3 & 1732 & 32 & 4 & 9 & 2 & 1 \\
\hline 1703 & 19 & 40 & 4 & 2 & 2 & 1733 & 39 & 1 & 7 & 4 & 2 \\
\hline 1704 & 55 & 4 & 5 & 2 & 2 & 1734 & 43 & 4 & 8 & 3 & 2 \\
\hline 1705 & 29 & 4 & 9 & 4 & 4 & 1735 & 37 & 3 & 8 & 3 & 4 \\
\hline 1706 & 40 & 8 & 2 & 0 & 0 & 1736 & 39 & 13 & 7 & 2 & 1 \\
\hline 1707 & 26 & 6 & 1 & 0 & 0 & 1737 & 34 & 4 & 8 & 5 & 2 \\
\hline 1708 & 27 & 20 & 11 & 3 & 1 & 1738 & 42 & 5 & 5 & 1 & 1 \\
\hline 1709 & 30 & 20 & 4 & 2 & 3 & 1739 & 42 & 8 & 8 & 3 & 0 \\
\hline 1700-09 & 332 & 131 & 52 & 14 & 15 & $1730-39$ & 385 & 54 & 70 & 29 & 13 \\
\hline 1710 & 36 & 12 & 2 & 0 & 0 & 1740 & 39 & 4 & 7 & 2 & 1 \\
\hline 1711 & 47 & 49 & 2 & 2 & 0 & 1741 & 38 & 8 & 7 & 2 & 2 \\
\hline 1712 & 19 & 29 & 3 & 2 & 1 & 1742 & 32 & 9 & 8 & 5 & 1 \\
\hline 1713 & 50 & 9 & 9 & 2 & 4 & 1743 & 35 & 2 & 5 & 4 & 1 \\
\hline 1714 & 30 & 13 & 6 & 2 & 3 & 1744 & 34 & 4 & 4 & 0 & 2 \\
\hline 1715 & 42 & 26 & 1 & 0 & 0 & 1745 & 31 & 12 & 3 & 0 & 0 \\
\hline 1716 & 22 & 9 & 4 & 1 & 0 & 1746 & 31 & 5 & 4 & 0 & 0 \\
\hline 1717 & 31 & 10 & 0 & 0 & 0 & 1747 & 29 & 2 & 4 & 0 & 1 \\
\hline 1718 & 19 & 11 & 2 & 1 & 1 & 1748 & 28 & 7 & 8 & 3 & 4 \\
\hline 1719 & 26 & 5 & 5 & 2 & 0 & 1749 & 38 & 13 & 8 & 3 & 4 \\
\hline $1710-19$ & 322 & 173 & 34 & 12 & 9 & $1740-49$ & 335 & 66 & 58 & 19 & 16 \\
\hline 1720 & 31 & 10 & 5 & 2 & 2 & 1750 & 30 & 6 & 8 & 2 & 4 \\
\hline 1721 & 33 & 10 & 7 & 4 & 0 & 1751 & 35 & 0 & 11 & 5 & 4 \\
\hline 1722 & 32 & 11 & 8 & 4 & 0 & 1752 & 39 & 0 & 5 & 3 & 0 \\
\hline 1723 & 35 & 13 & 6 & 4 & 2 & 1753 & 42 & 4 & 5 & 2 & 0 \\
\hline 1724 & 23 & 16 & 7 & 2 & 2 & 1754 & 35 & 2 & 2 & 0 & 0 \\
\hline 1725 & 30 & 10 & 2 & 2 & 1 & 1755 & 45 & 12 & 6 & 3 & 0 \\
\hline 1726 & 35 & 10 & 3 & 1 & 1 & 1756 & 37 & 10 & 10 & 5 & 0 \\
\hline 1727 & 38 & 10 & 6 & 3 & 3 & 1757 & 33 & 5 & 5 & 2 & 4 \\
\hline 1728 & 33 & 19 & 2 & 2 & 0 & 1758 & 35 & 14 & 3 & 3 & 2 \\
\hline 1729 & 35 & 13 & 7 & 5 & 1 & 1759 & 40 & 15 & 4 & 2 & 1 \\
\hline $1720-29$ & 325 & 122 & 53 & 29 & 12 & $1750-59$ & 371 & 68 & 59 & 27 & 15 \\
\hline
\end{tabular}


RIVIÈRE-OUELLE

\begin{tabular}{|c|c|c|c|c|c|c|c|c|c|c|c|}
\hline ANNÉES & B & MA & TM & ME & MV & ANNÉES & B & MA & $\mathbf{T M}$ & ME & $\mathbf{M V}$ \\
\hline 1700 & 16 & - & - & - & - & 1730 & 11 & - & 1 & - & - \\
\hline 1701 & 13 & - & 10 & 1 & - & 1731 & 11 & - & 1 & - & - \\
\hline 1702 & 32 & - & 4 & 2 & 1 & 1732 & 11 & - & 3 & 1 & - \\
\hline 1703 & 20 & 1 & 1 & - & - & 1733 & 19 & - & 3 & 2 & - \\
\hline 1704 & 26 & - & 4 & - & 1 & 1734 & 17 & - & 2 & - & - \\
\hline 1705 & & & & & & 1735 & 25 & 1 & 4 & 3 & 1 \\
\hline 1706 & 34 & 1 & 3 & - & - & 1736 & 18 & 3 & 5 & 1 & - \\
\hline 1707 & 27 & 2 & 5 & - & 1 & 1737 & 19 & 1 & 1 & 1 & - \\
\hline 1708 & 32 & 2 & 2 & - & - & 1738 & 20 & 1 & 2 & 2 & - \\
\hline 1709 & 27 & 5 & 3 & 2 & 1 & 1739 & 22 & 5 & 2 & 2 & - \\
\hline $1700-09$ & 227 & 11 & 32 & 5 & 4 & $1730-39$ & 173 & 11 & 24 & 12 & 1 \\
\hline 1710 & 26 & 2 & 7 & 1 & - & 1740 & 22 & 1 & 2 & 1 & - \\
\hline 1711 & 20 & 4 & 2 & 1 & 1 & 1741 & 14 & 3 & 3 & 3 & - \\
\hline 1712 & 26 & 5 & 6 & - & - & 1742 & 27 & 3 & 1 & - & - \\
\hline 1713 & 30 & - & 4 & - & 1 & 1743 & 21 & 2 & 3 & 3 & - \\
\hline 1714 & 23 & 2 & 3 & - & 1 & 1744 & 24 & 2 & 5 & 5 & - \\
\hline 1715 & & & & & & 1745 & 18 & 3 & 7 & 4 & - \\
\hline 1716 & 10 & 2 & 2 & 1 & 1 & 1746 & 24 & - & 4 & 2 & - \\
\hline 1717 & 7 & 3 & 1 & 1 & - & 1747 & 21 & 3 & 2 & 2 & - \\
\hline 1718 & 9 & - & 4 & 3 & - & 1748 & 24 & 1 & 5 & 3 & 1 \\
\hline 1719 & 5 & - & 2 & - & 1 & 1749 & 32 & 4 & 8 & 4 & - \\
\hline $1719-20$ & 156 & 18 & 31 & 7 & 5 & $1740-49$ & 227 & 22 & 40 & 27 & 1 \\
\hline 1720 & 12 & 1 & 4 & 1 & 1 & 1750 & 30 & - & 6 & 5 & 1 \\
\hline 1721 & 14 & 3 & 2 & 1 & - & 1751 & 28 & 8 & 4 & 2 & 1 \\
\hline 1722 & 13 & 2 & 1 & - & - & 1752 & 44 & 3 & 3 & 1 & - \\
\hline 1723 & 8 & 1 & 2 & - & - & 1753 & 32 & 5 & 7 & 2 & 1 \\
\hline 1724 & 6 & 2 & 1 & - & 2 & 1754 & 46 & 2 & 9 & 5 & 2 \\
\hline 1725 & 6 & 1 & 2 & 1 & - & 1755 & 47 & 7 & 3 & 2 & 1 \\
\hline 1726 & 9 & - & 3 & - & - & 1756 & 32 & 9 & 7 & 2 & 4 \\
\hline 1727 & 11 & - & 2 & - & - & 1757 & 55 & 2 & 13 & 6 & 5 \\
\hline 1728 & 6 & 1 & 2 & - & - & 1758 & 41 & 5 & 8 & 3 & 3 \\
\hline 1729 & 4 & 1 & 1 & - & - & 1759 & 48 & 11 & 1 & - & - \\
\hline $1720-29$ & 89 & 12 & 20 & 3 & 3 & $1750-59$ & 403 & 52 & 61 & 28 & 18 \\
\hline
\end{tabular}


SAINT-NICOLAS

\begin{tabular}{|c|c|c|c|c|c|c|c|c|c|c|c|}
\hline ANNÉES & B & MA & TM & ME & MV & ANNÉES & B & MA & TM & ME & MV \\
\hline 1700 & - & - & 1 & - & - & 1730 & 13 & 1 & 1 & 1 & - \\
\hline 1701 & 11 & 2 & - & 一 & - & 1731 & 19 & 5 & 4 & 3 & - \\
\hline 1702 & 2 & - & - & - & - & 1732 & 16 & 1 & 4 & 2 & 1 \\
\hline 1703 & 6 & 1 & 2 & 1 & - & 1733 & 16 & 1 & 3 & 3 & 1 \\
\hline 1704 & 9 & - & - & - & - & 1734 & 19 & 3 & 1 & 1 & - \\
\hline 1705 & 9 & - & 2 & 1 & - & 1735 & 24 & 2 & 3 & 1 & 2 \\
\hline 1706 & 16 & 2 & 2 & 1 & 1 & 1736 & 16 & 3 & 3 & 3 & 1 \\
\hline 1707 & 6 & - & 1 & - & - & 1737 & 23 & - & 1 & 1 & - \\
\hline 1708 & 12 & 5 & 1 & 1 & - & 1738 & 23 & 2 & 2 & - & - \\
\hline 1709 & 14 & 1 & 2 & 1 & - & 1739 & 17 & 1 & 3 & 2 & - \\
\hline $1700-09$ & 85 & 12 & 11 & 5 & 1 & $1730-39$ & 186 & 19 & 25 & 17 & 5 \\
\hline 1710 & 12 & 1 & 2 & 1 & - & 1740 & 18 & 1 & 1 & 1 & - \\
\hline 1711 & 15 & - & 1 & - & - & 1741 & 17 & 2 & 2 & 1 & 1 \\
\hline 1712 & 9 & 12 & 3 & 1 & - & 1742 & 19 & 5 & 5 & 4 & 1 \\
\hline 1713 & 15 & 2 & 2 & 2 & 1 & 1743 & 23 & 2 & 5 & 4 & 1 \\
\hline 1714 & 8 & - & 5 & 4 & 1 & 1744 & 16 & 5 & 2 & 1 & - \\
\hline 1715 & 15 & 3 & - & - & 2 & 1745 & 19 & - & 5 & 1 & 2 \\
\hline 1716 & 15 & 1 & 3 & - & - & 1746 & 17 & 4 & 1 & 1 & - \\
\hline 1717 & 11 & - & 2 & 1 & - & 1747 & 19 & 5 & 6 & 3 & 1 \\
\hline 1718 & 9 & 4 & 2 & - & - & 1748 & 21 & 4 & 9 & 5 & 2 \\
\hline 1719 & 15 & 1 & 3 & 1 & - & 1749 & 18 & 7 & 5 & 5 & 2 \\
\hline $1710-19$ & 124 & 24 & 23 & 10 & 4 & $1740-49$ & 187 & 35 & 41 & 26 & 10 \\
\hline 1720 & 7 & - & 1 & - & 1 & 1750 & 16 & 1 & 7 & 4 & - \\
\hline 1721 & 12 & - & 1 & - & 1 & 1751 & 21 & 3 & 6 & 2 & 2 \\
\hline 1722 & 6 & 1 & 2 & - & - & 1752 & 28 & 3 & 6 & 2 & 3 \\
\hline 1723 & 13 & 1 & 1 & - & - & 1753 & 20 & 5 & 3 & 1 & - \\
\hline 1724 & 11 & 1 & 2 & - & - & 1754 & 27 & 7 & 2 & 2 & - \\
\hline 1725 & 11 & 2 & 3 & 1 & - & 1755 & 17 & 4 & 2 & - & 1 \\
\hline 1726 & 7 & - & 3 & 1 & - & 1756 & 26 & 1 & 4 & 2 & 2 \\
\hline 1727 & 11 & 2 & 2 & 2 & - & 1757 & 21 & 6 & 13 & 3 & 3 \\
\hline 1728 & 13 & 1 & 4 & 3 & - & 1758 & 19 & 11 & 3 & - & 1 \\
\hline 1729 & 11 & 2 & 1 & - & - & 1759 & 17 & 2 & 2 & - & - \\
\hline $1720-29$ & 102 & 10 & 20 & 7 & 2 & $1750-59$ & 212 & 43 & 48 & 16 & 12 \\
\hline
\end{tabular}

